\title{
Fairness Control in Wavelength-Routed WDM Ring Networks
}

\author{
Kayvan Mosharaf, Ioannis Lambadaris, Jerome Talim, and Arash Shokrani \\ Department of Systems and Computer Engineering, Carleton University, Ottawa, Canada \\ Email: \{mosharaf,jtalim,ioannis,shokrani\}@ sce.carleton.ca
}

\begin{abstract}
We investigate a threshold-based wavelength allocation scheme in order to support fairness and service differentiation in WDM unidirectional ring networks. A ring network can handle different classes of traffic streams which differ by their hop-counts (i.e., the number of hops used from source to destination). We assume that for each class of traffic, call interarrival and holding times are exponentially distributed. In such a network, classes of calls with smaller hop-counts, experience lower blocking rates than ones with greater hopcounts. In this paper, a Multi-Threshold wavelength allocation scheme is proposed to provide equal blocking probabilities experienced by different classes. A recursive simulation-based algorithm is designed to numerically compute the optimal thresholds. Simulation results compare the performance of our proposed scheme, with that of Complete Sharing and Complete Partitioning schemes.
\end{abstract}

\section{INTRODUCTION}

Without doubt Wavelength Division Multiplexing (WDM) is a promising candidate to handle the bandwidth demand of future Wide Area Networks. In wavelength routing networks, each optical path must be established with a specific wavelength between each source-destination pair. This is known as wavelength continuity constraint and can be relaxed by using Wavelength Converters (WC) at intermediate nodes. The Routing and Wavelength Assignment (RWA) problem is an important issue in WDM networks. RWA is usually divided into two separate sub-problems: wavelength assignment problem, and routing problem. Many heuristic algorithms such as First-Fit, Most-Used, Max-Sum and Random wavelength assignment have already been proposed [1]. The objective of these algorithms is typically to minimize the overall call blocking probability or maximize the overall utilization in a single-class network. Some analytical studies related to this problem can be found in [2], [3].

Threshold-based RWA schemes are introduced for dynamic wavelength-routed mesh networks in [4], [5]. In [4], a basic routing method such as Fixed Routing (FR) and Fixed Alternative Routing (FAR), combined with a threshold-based allocation technique, is used in order to obtain the same grade of service on a multi-hop route as on a single-hop. In [5], a routing and signaling protocol called Asynchronous Criticality Avoidance (ACA) is developed to improve the blocking probabilities in mesh networks. ACA mechanism marks a set of wavelength channels as critical if the available channels between a source-destination pair is less than a predefined threshold. The critical channels information is announced to the other nodes in the network so that the other sourcedestination pairs avoid the use of these critical channels [5]. In this paper, we will design a threshold-based RWA scheme to improve fairness in wavelength-routed WDM ring networks.

The purpose of this paper is to develop a wavelength allocation mechanism to support fairness and service differentiation in WDM ring networks. We first define a Multi-Threshold (MT) wavelength allocation policy which can equalize the class-based blocking probabilities in an unidirectional WDM ring network. To determine the optimal thresholds of MT policy, we then define an objective function to be minimized. Using the properties of the objective function, a recursive algorithm is developed to find the optimal thresholds.

The rest of the paper is organized as follows. In Section II, we describe the network model and make some assumptions about the network. In Section III, we introduce the MT allocation scheme and define an objective function which measures the differences between class-based blocking probabilities. Section IV describes our recursive simulation-based optimization algorithm. Section V compares the performance of the proposed algorithm with other standard algorithms. Conclusions are presented in Section VI.

\section{NeTwORK MODEL}

We consider a unidirectional WDM ring network with $N$ nodes. Each node is equipped by Optical Add/Drop Multiplexers (OADM). Each link carries one fiber with $W$ wavelengths. The network includes $N(N-1)$ classes of traffic streams characterized by their source-destination. A call from Class $C_{h}^{r}, r=1,2, \ldots, N$ and $h=1,2, \ldots, N-1$, originates at node $r$ and passes through $h$ hops from origin to destination. We assume that classes with the same hop-count (i.e., the number of hops used from origin to destination) are assigned the same weighting factor (priority), and the same arrival rate (i.e., $\lambda_{C_{h}^{1}}=\lambda_{C_{h}^{2}}=\ldots=\lambda_{C_{h}^{N}}, h=1,2, \ldots, N-1$, where $h$ is the hop-count). Therefore, we can merge classes with the same hop-count into a single class. In the following, we refer to classes with hop-count $h$ as Class $c_{h}$. We consider two types of networks in terms of WC usage: networks with WCs in all nodes and network without any WC. In a WDM network without WCs, wavelength continuity constraint is considered.

We also make the following assumptions.

- Arrivals of class $c_{h}$ calls are distributed according to a Poisson process with rate $\lambda_{h}$. Calls of class $c_{h}$ are uniformly distributed among nodes. The call holding time 
of this class is exponentially distributed with mean $\mu_{h}^{-1}$. Unless otherwise stated, we assume $\mu_{h}^{-1}=1$.

- The total offer load per link is $\rho_{L}=\sum_{h=1}^{N-1}\left(h \lambda_{h} / \mu_{h}\right)$.

- Any arriving call from class $c_{h}$ is blocked when no wavelength is available along its path.

- Blocked calls do not interfere with the system.

In such a system, since calls from different classes compete for access to the network, fairness management and resource sharing control become very challenging issues. Complete Sharing (CS) and Complete Partitioning (CP) algorithms are two of the well-known capacity access control schemes [6]. These two are briefly outlined and compared in the following paragraphs.

CS policy does not reserve resources such as bandwidth or wavelength to any class of calls. In addition, an arriving call is accepted if at least one wavelength is free to carry that call. In this case, calls with smaller hop counts (respectively, higher arrival rates) may starve calls with greater hop-counts (respectively, lower arrival rates). Although the global network utilization is high in this case, the system is unfair in the sense that there is a noticeable difference between the blocking probabilities experienced by different classes of users. Here, the objective of fairness is to keep this difference as small as possible, so that all classes with different hop-counts experience the same blocking probability.

On the other hand, when CP policy is implemented, each class is dedicated a portion of the resources that cannot be used by calls from other classes. Hence, it supports service differentiation and controls class-based blocking probabilities. However, CP policy may not maximize the overall utilization of the available resources.

Our goal, then, is to design a threshold-based allocation mechanism which achieves the fairness objective and maximizes overall utilization. In the following sections, we introduce a Multi-Threshold allocation policy to improve fairness in a WDM ring network.

\section{Multi-Threshold Wavelength Allocation}

As mentioned earlier in Section II, CS policy provides high utilization and poor service differentiation (in particular, fairness) whereas CP achieves the fairness but the overall utilization deteriorates. This reduction in utilization results directly from putting up barriers against sharing wavelengths.

In this section, we propose a Multi-Threshold (MT) allocation scheme which takes advantage of both $\mathrm{CP}$ and CS policies. When MT policy is used, while all classes of users share the wavelengths, classes with higher hop-counts are protected from the ones with lower hop-counts on each link of the ring network.

We define $\boldsymbol{\tau}=\left(\tau_{1}, \tau_{2}, \ldots, \tau_{N-1}\right)$ a vector representing the thresholds of MT allocation where $\tau_{i}, i=1,2, \ldots, N-1$, is the threshold associated with class $c_{i}$ traffic on each link of the ring network. To ensure that calls with higher hop-counts are protected from the ones with lower hop-counts, we assume that $\tau_{1} \geq \tau_{2} \geq \ldots \geq \tau_{N-1}$. Since calls from class $c_{N-1}$ experience the highest blocking probability in the ring, they are always

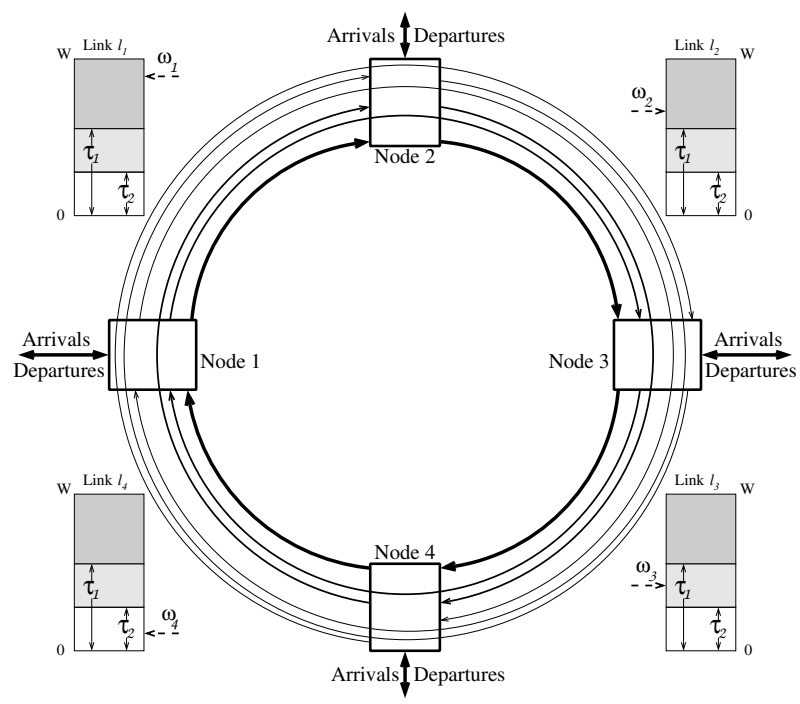

Fig. 1. A 4-node unidirectional ring network.

accepted in the system. As a result, we set $\tau_{N-1}=0$. Let $l_{n}$, $n=1,2, \ldots, N$, be the link between node $n$ and node $n+1$ and $\omega_{n}$ denote the number of available wavelengths on link $l_{n}$. An arriving call of class $c_{i}$ will be assigned an available wavelength only if $\omega_{n}$ on all the links along its path is greater than or equal to $\tau_{i}$.

As an example, consider a 4-node unidirectional ring network shown in Fig. 1. One can note that on each link, the total number of available wavelengths $W$ is partitioned into three sets. When the number of free wavelengths on a link is greater or equal to threshold $\tau_{1}$, then all three classes of calls share the available wavelengths on that link. If this number is between $\tau_{1}$ and $\tau_{2}$, then only class $c_{1}$ calls will be dropped. Furthermore, if the number of free wavelengths is less than $\tau_{2}$ on a link, then both $c_{1}$ and $c_{2}$ calls will be blocked and $c_{3}$ calls are the only ones allowed to use the wavelength resources on that particular link. As shown in Fig. 1, available wavelengths on links $l_{1}$ and $l_{2}$ are greater than $\tau_{1}$. Thus, all classes of calls which pass through $l_{1}$ and $l_{2}$ can occupy free wavelengths of these links. Since $\tau_{2}<\omega_{3}<\tau_{1}$, only $c_{1}$ calls, which use link $l_{3}$ as the part of their route, will be blocked. On links $l_{4}$, we have $\omega_{4}<\tau_{2}$ and as a result, only $c_{3}$ calls are allowed to occupy an idle wavelength on this link.

The next step towards MT allocation implementation is to determine the threshold vector $\tau$, so that all classes of calls obtain the same Grade of Service $(\mathrm{GoS})$ in terms of blocking probabilities. To do so, we define the following objective function

$$
f(\boldsymbol{\tau})=\sum_{i=1}^{N-2} \sum_{j=i+1}^{N-1}\left|B_{i}(\boldsymbol{\tau})-B_{j}(\boldsymbol{\tau})\right|
$$

where $B_{i}(\boldsymbol{\tau})$ is the class $c_{i}$ blocking probability associated with $\boldsymbol{\tau}$. To determine $B_{i}(\boldsymbol{\tau})$, we have developed a simulation program in OPNET. In order to obtain an accurate value of $f$, the simulation experiment continues until the steady- 


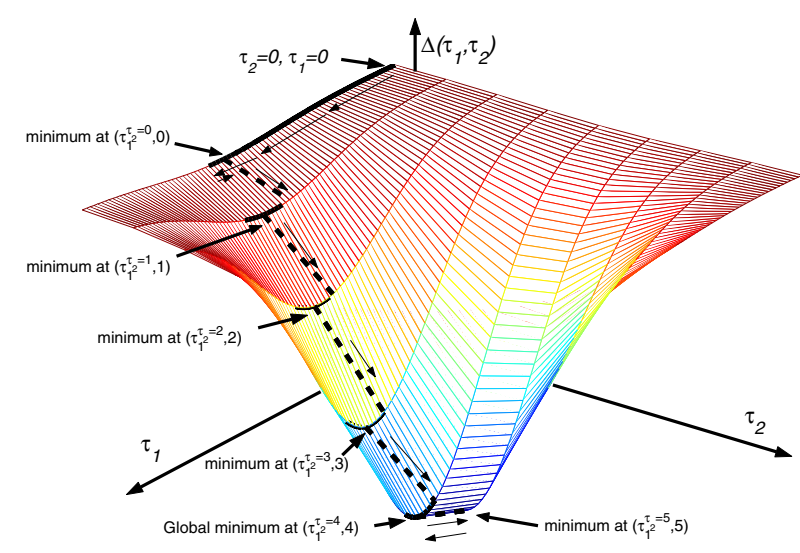

Fig. 2. An illustrative example to show the minimization algorithm for a function with one unique global minimum.

state regime is reached. Since this objective function measures the differences between class-based blocking probabilities, by minimizing that, we can achieve fairness. In the following section, we develop an efficient algorithm to minimize the objective function and determine the optimal $\tau$

\section{Recursive Optimization Algorithm}

The optimization procedure can take advantage of structural properties of the objective function (such as monotonicity or multimodularity) to improve the computation time [7]. In Theorem 1, we prove that the objective function $f$ has only one unique minimum and it does not have local minima. According to this property and the fact that $W \geq \tau_{1} \geq \tau_{2} \geq \ldots \geq \tau_{N-1} \geq$ 0 , we propose a gradient-like optimization algorithm in order to determine the appropriate values for the thresholds.

Definition 1: A function is unimodal if it decreases to a minimum and then increases. A unimodal function has a single minimum.

Theorem 1: The objective function $f$ is unimodal.

Proof: Because of space limitation, the proof is given in [8].

Fig. 2 depicts an illustrative example of the objective function, $f\left(\tau_{1}, \tau_{2}\right)$, as a function of $\tau_{1}$ and $\tau_{2}$. To find the global minimum, one can first search for a minimum when $\tau_{2}=0$. This is done by incrementally increasing $\tau_{1}$. After finding the optimal $\tau_{1}$, denoted by $\tau_{1}^{\tau_{2}=0}$, and reaching the minimum at $\left(\tau_{1}^{\tau_{2}=0}, 0\right)$, we increase $\tau_{2}$ by 1 and search for another minimum in the neighborhood of $\left(\tau_{1}^{\tau_{2}=0}, 1\right)$. As can be seen in Fig. 2, we again need to increase $\tau_{1}^{\tau_{2}=0}$ to reach a minimum at $\left(\tau_{1}^{\tau_{2}=1}, 1\right)$. The reason is that at point $\left(\tau_{1}^{\tau_{2}=0}, 1\right)$, decreasing of $\tau_{1}$ results in increasing of $f$ and as a result, we need to increase $\tau_{1}$ in order to get to the minimum. This procedure continues until the global minimum is reached at point $\left(\tau_{1}^{\tau_{2}=4}, 4\right)$. In [8], we also show that the following inequality is satisfied

$$
\tau_{1}^{\tau_{2}=4} \geq \tau_{1}^{\tau_{2}=3} \geq \tau_{1}^{\tau_{2}=2} \geq \tau_{1}^{\tau_{2}=1} \geq \tau_{1}^{\tau_{2}=0}
$$

We generalize our search for $N-1$ classes as follows.

Let $\boldsymbol{\tau}_{\tau_{i+1}, \ldots, \tau_{N-1}}^{i}=\left(\tau_{1}^{*_{i}}, \tau_{2}^{*_{i}}, \ldots, \tau_{i}^{*_{i}}, \tau_{i+1}, \ldots, \tau_{N-1}\right)$ be the optimal value of $\tau$ when the last $N-i-1$ thresholds are fixed. In other words

$$
\boldsymbol{\tau}_{\tau_{i+1}, \ldots, \tau_{N-1}}^{i}=\arg \min _{\tau_{1}, \tau_{2}, \cdots, \tau_{i}} f\left(\tau_{1}, \cdots, \tau_{i}, \cdots, \tau_{N-1}\right)
$$

For simplicity, index of $\boldsymbol{\tau}_{\tau_{i+1}, \ldots, \tau_{N-1}}^{i}$ is no longer mentioned in notation. In the following, we introduce a recursive algorithm, $\Theta_{i+1}$, which receives $\tau^{i}$ and computes $\tau^{i+1}$.

$$
\Theta_{i+1}\left(\boldsymbol{\tau}^{i}\right)=\boldsymbol{\tau}^{i+1}
$$

Clearly, by using an initial threshold vector $\tau^{0}$ and running this algorithm for $N-1$ times, we can obtain $\tau^{N-1}$. Similar to inequality (2), we have shown [8] that when we start our search from initial value $\tau^{0}=(0,0, \ldots, 0)$, then

$$
\tau_{1}^{*_{i+1}} \geq \tau_{1}^{*_{i}}, \tau_{2}^{*_{i+1}} \geq \tau_{2}^{*_{i}}, \cdots, \tau_{i}^{*_{i+1}} \geq \tau_{i}^{*_{i}}
$$

Because of this important property, if the initial value is $\boldsymbol{\tau}^{0}=(0,0, \ldots, 0)$, we only need to increase $\tau_{i}$ to find the minimum. Fig. 3 depicts the block diagram of this recursive algorithm in the $(i+1)^{\text {th }}$ recursion.

As can be seen, $\Theta_{i+1}$ is expressed based on $\Theta_{i}$, the algorithm's $i^{\text {th }}$ recursion. Note that $\Theta_{0}$ is an identity function (i.e., the input and output of $\Theta_{0}$ are the same, $\Theta_{0}(\tau)=\tau$ ). By initializing the recursive algorithm with $\tau^{0}=(0,0, \ldots, 0)$, we can recursively compute the optimal threshold vector $\tau^{N-1}$. For instance, in the first recursion where $\tau=\tau^{0}=$ $(0,0, \ldots, 0)$, we increase $\tau_{1}$ until a minimum is reached at point $\boldsymbol{\tau}^{1}=\left(\tau_{1}^{*_{1}}, 0, \ldots, 0\right)$. In the second recursion where $\boldsymbol{\tau}=\boldsymbol{\tau}^{1}$, we first increase $\tau_{2}$ and then for each value of $\tau_{2}$, we apply $\Theta_{1}$ in order to find a minimum for that particular $\tau_{2}$. Increasing $\tau_{2}$ and applying $\Theta_{1}$ continues until a minimum is found at point $\tau^{2}=\left(\tau_{1}^{*_{2}}, \tau_{2}^{*_{2}}, \ldots, 0\right)$. Recursively, the global minimum and the associated optimal threshold vector $\tau^{N-1}$ will be determined.

More precisely, this optimization problem can be solved by the following procedure.

Step 1: Set $i=0$ and $\tau_{1}^{*_{i}}, \tau_{2}^{*_{i}}, \ldots, \tau_{i}^{*_{i}}, \ldots, \tau_{N-1}^{*_{i}}=0$.

Step 2: Set $\tau^{i}=\left(\tau_{1}^{*_{i}}, \tau_{2}^{*_{i}}, \ldots, \tau_{i}^{*_{i}}, \ldots, \tau_{N-1}^{*_{i}}\right)$.

Step 3: Use $\tau^{i}$ as the input of $\Theta_{i+1}$ and compute $\tau^{i+1}$.

Step 4: If $\tau^{i+1}=\tau^{i}$, then stop the recursion. $\tau^{i}$ is the optimal value. Otherwise, $i=i+1$ and go to Step 2 .

We implemented the above procedure for a 4-node 40wavelength unidirectional ring network without WCs for $\lambda_{1}=2 \lambda_{2}=3 \lambda_{3}=30$. To illustrate our recursive simulationbased algorithm, Fig. 4 shows the values of $\tau_{1}, \tau_{2}, B_{1}, B_{2}$ and $B_{3}$ in different steps of the procedure. In order to calculate an accurate $f$, the simulation continues for 500,000 arrivals

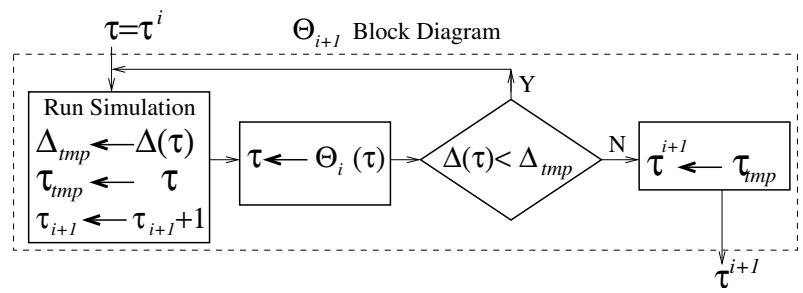

Fig. 3. Block Diagram of the recursive minimization function $\Theta_{i+1}$. 


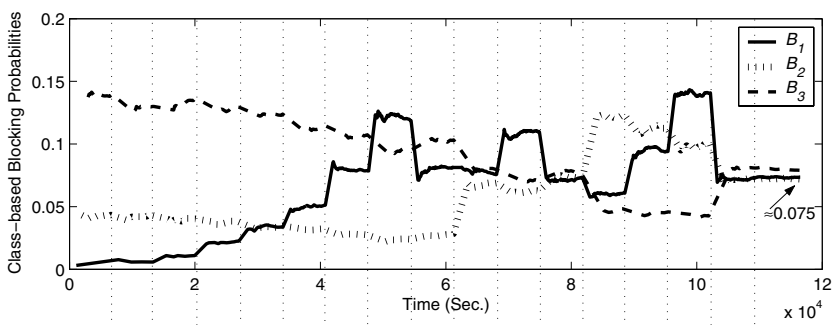

$500,0.00$ arrivals

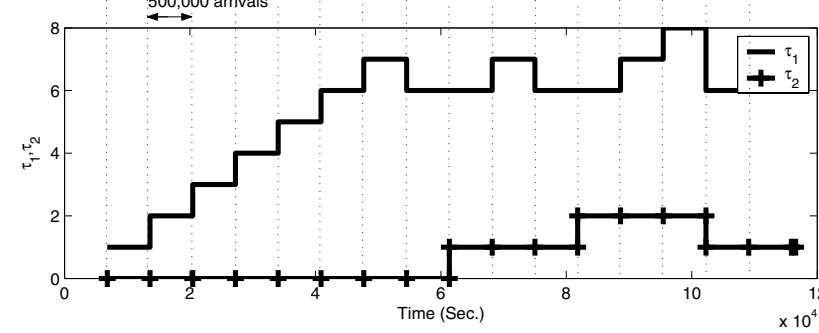

Fig. 4. Evaluation of $\tau_{1}$ and $\tau_{2}$ according to the recursive simulation-based algorithm for a 4-node 40-wavelength ring network without WCs. Between two consecutive dot-lines 500,000 arrivals are generated.

in each run. As it is presented, after a few steps the algorithm converges and we get $\tau^{3}=(6,1,0)$. After applying MT allocation scheme with the optimal thresholds, all three classes of users gain almost the same GoS, $B_{1} \approx B_{2} \approx B_{3} \approx 0.075$.

\section{Performance Comparison}

In this section, we compare the performance of our proposed wavelength allocation scheme, with $\mathrm{CS}$ and $\mathrm{CP}$ policies. The simulation results are carried out for 4-node and 8-node unidirectional ring networks with and without WCs. The performance metric used in the numerical comparison deals with fairness and blocking probabilities.

Suppose that $c_{i}$ and $c_{j}$ calls experience the highest and lowest blocking probabilities, respectively, then we define fairness ratio as $f_{r}:=B_{i} / B_{j}$. The closer the $f_{r}$ to 1 , the better the fairness is. Also in our simulations, the arrival rate of class $c_{i}$ is set inversely proportional to its hop-count (i.e., $\left.\lambda_{1}=2 \lambda_{2}=\ldots=(N-1) \lambda_{N-1}\right)$. Hence, on each link, the expected wavelength request rate of each class is the same.

In order to employ $\mathrm{CP}$ policy, one can partition the total number of available wavelengths into separate sets, each of which is dedicated to one of the origin-destination pairs. Note that non-overlapping origin-destination pairs can share the same set of wavelengths. For example, consider the 4-node ring network depicted in Fig. 1. Since class $c_{1}$ calls do not share any link, they can use the same set of wavelengths. Class $c_{2}$ calls passing through $\left\{l_{1}, l_{2}\right\}$ and also $c_{2}$ calls using $\left\{l_{3}, l_{4}\right\}$ can share the same set of wavelengths. Similarly, $c_{2}$ calls using $\left\{l_{2}, l_{3}\right\}$ and $c_{2}$ calls passing through $\left\{l_{4}, l_{1}\right\}$ can use one set. Four different sets are required for overlapping $c_{3}$ calls. As a result, the total wavelengths is partitioned into seven sets; set $\mathcal{S}_{1}$ is dedicated to class $c_{1}$ calls, set $\mathcal{S}_{2}$ is assigned to $c_{2}$ calls passing through $\left\{l_{1}, l_{2}\right\}$ and $\left\{l_{3}, l_{4}\right\}$. Set $\mathcal{S}_{3}$ is allocated to $c_{2}$ calls using $\left\{l_{2}, l_{3}\right\}$ and $\left\{l_{4}, l_{1}\right\}$. Four sets, $\mathcal{S}_{4}$ through $\mathcal{S}_{7}$, are needed for $c_{3}$ calls. Let $m_{j}$ denote the number of wavelengths
TABLE I

CLASS-BASED BLOCKING PROBABILITIES AND FAIRNESS RATIOS FOR A 4-NODE 40-WAVELENGTH RING NETWORK WITHOUT WCS.

\begin{tabular}{|c|c|ccc|c|c|}
\hline$\rho$ & Policy & $B_{1}$ & $B_{2}$ & $B_{3}$ & $f_{r}$ & $\boldsymbol{\tau}^{3}$ \\
\hline 20 & CS & .000008 & 0.00021 & 0.00064 & 80.0 & - \\
20 & MT & 0.00049 & 0.00045 & 0.00043 & $\mathbf{1 . 1 4}$ & $(4,1,0)$ \\
25 & CS & 0.00033 & 0.00795 & 0.02473 & 75.0 & - \\
25 & MT & 0.01410 & 0.01561 & 0.01505 & $\mathbf{1 . 1 0}$ & $(5,1,0)$ \\
30 & CS & 0.00201 & 0.04344 & 0.13807 & 67.7 & - \\
30 & MT & 0.07360 & 0.07063 & 0.07750 & $\mathbf{1 . 1 0}$ & $(6,1,0)$ \\
35 & CS & 0.00502 & 0.10198 & 0.29933 & 59.6 & - \\
35 & MT & 0.13509 & 0.15496 & 0.17947 & $\mathbf{1 . 3 2}$ & $(6,1,0)$ \\
40 & CS & 0.00859 & 0.13096 & 0.37806 & 44.0 & - \\
40 & MT & 0.16722 & 0.16821 & 0.19403 & $\mathbf{1 . 1 6}$ & $(6,1,0)$ \\
\hline
\end{tabular}

in set $\mathcal{S}_{j}$. Note that all sets associated with class $c_{i}$ have the same number of wavelengths. Therefore, we have $m_{2}=m_{3}$ and $m_{4}=m_{5}=m_{6}=m_{7}$ and $W=\sum_{j=1}^{7} m_{j}$.

Using Erlang's B formula, we can compute $p_{j}$, the blocking probability of a call which uses wavelengths of set $\mathcal{S}_{j}$ by

$$
p_{j}=\frac{\frac{1}{m_{j} !}\left(\frac{\lambda}{\mu}\right)^{m_{j}}}{1+\sum_{n=1}^{m_{j}} \frac{1}{n !}\left(\frac{\lambda}{\mu}\right)^{n}}
$$

where $\lambda / \mu$ is traffic density to wavelengths of $\mathcal{S}_{j}$. We can find appropriate values for $m_{j}$ in a way that $p_{1}=p_{2}=\ldots=p_{7}$.

We simulate the system by deploying the recursive algorithm implemented in Section IV to calculate the performance metric of MT allocation policy. For implementing CP, the above procedure is used. Finally, the system is simulated without any allocation policy to find the performance of CS.

We first study the fairness objective in the ring network shown in Fig. 1 when MT, CP and CS policies are used. Table I shows the class-based blocking probabilities for a 40wavelength ring network without WCs. As Table I reports, deploying MT policy results in a fairness ratio close to 1 . As expected, when CS policy is used the fairness ratio is high, $f_{r} \in[44,80]$. For low traffic load, the blocking rates are relatively small and the competition for access to the network is low. Conversely, when the offered load is high, calls from different classes compete for utilizing resources. As a result, by increasing traffic load, $\tau_{1}^{*_{3}}$ increases to block more $c_{1}$ calls in order to protect $c_{2}$ and $c_{3}$ calls .

Now we compare MT and CP policies. This comparison is based on the number of wavelengths required to provide a certain GoS. By using (6), we computed $m_{1}=13, m_{2}=m_{3}=$ 8 and $m_{4}=m_{5}=m_{6}=m_{7}=6$. Therefore, 53 wavelengths $(W=13+2 \times 8+4 \times 6)$ is required for $\mathrm{CP}$ implementation, which shows $32.5 \%$ growth in terms of network resource cost. For instance when $\rho=30$ and CP is used, we get $f_{r}=1.2$ and $B_{1}=0.084, B_{2}=0.070$ and $B_{3}=0.072$.

We also investigate the impact of using WCs on fairness. Table II presents the performance of MT and CS policies for the above example when all nodes are equipped by WCs. As reported in this table, even if MT policy is not used, utilizing WCs considerably improves the fairness ratio.

Comparison of Tables I and II illustrates that: 1) for systems without WCs, using MT allocation policy results in a very 
TABLE II

CLASS-BASED BLOCKING PROBABILITIES AND FAIRNESS RATIOS FOR A 4-NODE 40-WAVELENGTH RING NETWORK WITH WCS IN ALL NODES.

\begin{tabular}{|c|c|ccc|c|c|}
\hline$\rho$ & Policy & $B_{1}$ & $B_{2}$ & $B_{3}$ & $f_{r}$ & $\boldsymbol{\tau}^{3}$ \\
\hline 25 & CS & 0.00137 & 0.00257 & 0.00400 & 2.91 & - \\
25 & MT & 0.00280 & 0.00156 & 0.00259 & $\mathbf{1 . 7 9}$ & $(1,0,0)$ \\
30 & CS & 0.01245 & 0.02361 & 0.03522 & 2.82 & - \\
30 & MT & 0.02547 & 0.01609 & 0.02425 & $\mathbf{1 . 5 8}$ & $(1,0,0)$ \\
35 & CS & 0.03990 & 0.07491 & 0.10968 & 2.74 & - \\
35 & MT & 0.07935 & 0.05349 & 0.07911 & $\mathbf{1 . 4 8}$ & $(1,0,0)$ \\
40 & CS & 0.07695 & 0.14370 & 0.20794 & 2.70 & - \\
40 & MT & 0.15311 & 0.10807 & 0.15417 & $\mathbf{1 . 4 2}$ & $(1,0,0)$ \\
45 & CS & 0.11677 & 0.21677 & 0.30387 & 2.60 & - \\
45 & MT & 0.22698 & 0.16142 & 0.23046 & $\mathbf{1 . 4 2}$ & $(1,0,0)$ \\
\hline \hline
\end{tabular}

good fairness ratio (i.e., $f_{r} \approx 1$ ). 2) Deploying WCs not only decreases the overall blocking probabilities but also improves the fairness ratio. For example, the overall blocking rate of the system using MT allocation without WCs is equal to 0.0733 for $\rho=30$ whereas this metric for the network with WCs is 0.0226. 3) In both cases, with and without WCs, MT allocation mechanism is able to improve the fairness ratio. One can see that $f_{r} \in[1.10,1.16]$ and $f_{r} \in[1.42,1.78]$ for the networks with and without WCs, respectively. 4) The optimal thresholds in the network with WCs are smaller than the ones in the network without WCs.

We now implement and study the performance of MT and CS policies in a 8-node 110-wavelength unidirectional WDM ring network. Table III (respectively, IV) presents the classbased blocking probabilities and fairness ratios of the system with WCs (respectively, without WCs). It can be observed from Table III that when CS is deployed the fairness ratio is above 1000 for all traffic loads. When MT allocation is implemented, the fairness ratio is remarkably improved, $f_{r} \in$ $[1.39,1.77]$. As Table IV shows, using WCs in the system plays an important role in fairness improvement. Similar to the 4-node network case, Table III confirms that in order to preserve wavelengths for calls with higher hop-counts, $\tau_{1}^{* 7}$ and $\tau_{2}^{* 7}$ will increase when the offered load increases. Comparison of Tables I and III shows that when the number of nodes increases in ring networks without WCs, using MT allocation scheme becomes more crucial.

According to the above numerical results, MT wavelength allocation scheme may block one class of users to preserve

TABLE III

CLASS-BASED BLOCKING PROBABILITIES AND FAIRNESS RATIOS FOR A 8-NODE 110-WAVELENGTH RING NETWORK WITHOUT WCS.

\begin{tabular}{|l|cc|cc|cc|}
\hline & \multicolumn{2}{|c|}{$\rho=600$} & \multicolumn{2}{c|}{$\rho=700$} & \multicolumn{2}{c|}{$\rho=800$} \\
\cline { 2 - 7 } & $\mathrm{CS}$ & $\mathrm{MT}$ & $\mathrm{CS}$ & $\mathrm{MT}$ & $\mathrm{CS}$ & $\mathrm{MT}$ \\
\hline$B_{1}$ & .00000 & .01450 & .00000 & .07205 & .00000 & .16216 \\
$B_{2}$ & .00000 & .00847 & .00000 & .06887 & .00080 & .12974 \\
$B_{3}$ & .00071 & .01423 & .00688 & .08841 & .01715 & .17925 \\
$B_{4}$ & .00518 & .01098 & .04304 & .06650 & .10057 & .15347 \\
$B_{5}$ & .01326 & .00819 & .10927 & .06420 & .24370 & .14202 \\
$B_{6}$ & .01887 & .01137 & .15298 & .08567 & .33907 & .18776 \\
$B_{7}$ & .02030 & .01241 & .16109 & .08973 & .36291 & .20718 \\
\hline$f_{r}$ & $>1000$ & $\mathbf{1 . 7 7}$ & $>1000$ & $\mathbf{1 . 3 9}$ & $>1000$ & $\mathbf{1 . 5 9}$ \\
\hline $\boldsymbol{\tau}^{7}$ & $(19,8,4,1,0,0,0)$ & \multicolumn{2}{|c|}{$(20,9,4,1,0,0,0)$} & \multicolumn{2}{|c|}{$(21,9,4,1,0,0,0)$} \\
\hline \hline
\end{tabular}

TABLE IV

CLASS-BASED BLOCKING PROBABILITIES AND FAIRNESS RATIOS FOR A 8-NODE 110-WAVELENGTH RING NETWORK WITH WCS IN ALL NODES.

\begin{tabular}{|l|cc|cc|cc|}
\hline & \multicolumn{2}{|c|}{$\rho=700$} & \multicolumn{2}{|c|}{$\rho=800$} & \multicolumn{2}{c|}{$\rho=900$} \\
\cline { 2 - 7 } & $\mathrm{CS}$ & $\mathrm{MT}$ & $\mathrm{CS}$ & $\mathrm{MT}$ & $\mathrm{CS}$ & $\mathrm{MT}$ \\
\hline$B_{1}$ & .00192 & .00617 & .01388 & .04728 & .03271 & .11702 \\
$B_{2}$ & .00387 & .00633 & .02617 & .04705 & .06183 & .11821 \\
$B_{3}$ & .00567 & .00341 & .03941 & .02665 & .09055 & .06720 \\
$B_{4}$ & .00749 & .00474 & .05026 & .03476 & .11968 & .08634 \\
$B_{5}$ & .00886 & .00598 & .06159 & .04172 & .14601 & .10720 \\
$B_{6}$ & .01037 & .00672 & .07388 & .05029 & .17114 & .12922 \\
$B_{7}$ & .01210 & .00849 & .08473 & .05841 & .19707 & .14428 \\
\hline$f_{r}$ & 6.30 & 2.49 & 6.10 & 2.19 & 6.02 & 2.14 \\
\hline $\boldsymbol{\tau}^{7}$ & \multicolumn{2}{|c|}{$(2,1,0,0,0,0,0)$} & \multicolumn{2}{|c|}{$(2,1,0,0,0,0,0)$} & \multicolumn{2}{|c|}{$(2,1,0,0,0,0,0)$} \\
\hline \hline
\end{tabular}

wavelengths for other classes. In fact, we reduce the blocking rates of calls with higher hop-counts at the expense of increasing the blocking rates of ones with lower hop-counts .

\section{CONCLUSION}

Our contribution in this paper is to develop a thresholdbased wavelength allocation scheme to provide service differentiation and fairness control in WDM ring networks. To determine the optimal thresholds of our allocation scheme, we defined a objective function to be minimized. Using some properties of the objective function, we proposed and implemented a recursive simulation-based algorithm to find the optimal thresholds. Simulation results compared the performance of the proposed approach, to ones of CS and CP policies. It is observed that we can achieve substantial improvement in terms of fairness.

\section{REFERENCES}

[1] H. Zang, J. P. Jue, and B. Mukherjee, "A Review of Routing and Wavelength Assignment Approaches for Wavelength-Routed Optical WDM Networks," Optical Networks Magazine, vol. 1, no. 1, Jan. 2000, pp. 47-60

[2] R. A. Barry and P. A. Humblet, "Models of Blocking Probability in All-Optical Networks with and without Wavelength Changers," IEEE J. Sel. Areas Comm., vol. 14,no. 5, June 1996, pp. 858-867.

[3] Y. Zhu, G. N. Rouskas and H. G. Perros, "A Path Decomposition Approach for Computing Blocking Probabilities in Wavelength-Routing Networks," IEEE/ACM Trans. on Net., vol. 8, No. 6, Dec. 2000, pp. 747-762.

[4] A. Birman and A. Kershenbaum, "Routing and Wavelength Assignment Methods in Single-Hop All-Optical Networks with Blocking," in Proc. of IEEE INFOCOM '95, vol. 2, April 1995, pp. 431-438.

[5] Pin-Han Ho and H.T. Mouftah, "A Novel Distributed Control Protocol in Dynamic Wavelength-routed Optical Networks," IEEE Communications Magazine, vol. 40 , issue 11 , Nov. 2002, pp. 38-45.

[6] K. W. Ross, D. H. K. Tsang, "The Stochastic Knapsack Problem," IEEE Transactions on Communications, vol. 37, no. 7, Jul. 1989, pp. 740-747.

[7] E. Altman, B. Gaujal and A. Hordijk "Multimodularity, Convexity and Optimization Properties," Math. of Oper. Research, Volume 25, pp. 324-347, 2000

[8] K. Mosharaf, J. Talim and I. Lambadaris, "Multi-Threshold Wavelength Allocation Policy for Fairness Control in WDM Ring Networks," Carleton University Technical Report, SCE-04-13, Aug. 2004. 